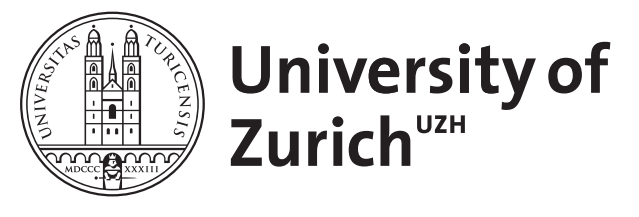

\title{
Magnetresonanztomographie der Schulter
}

\author{
Zanetti, M ; Saupe, N
}

\begin{abstract}
Die MRT der Schulter spielt neben Anamnese und klinischer Untersuchung eine bedeutende Rolle bei der Diagnose und Differenzierung zahlreicher Schulterpathologien und der Abklärung chronischer Schulterschmerzen. Wichtige Indikationsgebiete der MRT sind die Evaluation der Rotatorenmanschette und des Labrum-Kapsel-Apparats. Besondere Bedeutung hat die Beurteilung der Rotatorenmanschettenmuskulatur. Die immer noch kontrovers diskutierte MR-Arthrographie zeigt ihr größtes Potenzial bei der präzisen Unterscheidung von kleinen Rotatorenmanschettenläsionen, Labrum-Kapsel-Läsionen und Läsionen der langen Bizepssehne. In dem Beitrag wird auf die häufigsten pathologischen Veränderungen des Schultergelenks eingegangen. Daneben wird gezeigt, wie die Befunde für Schulterorthopäden beschrieben und quantifiziert werden
\end{abstract}

DOI: https://doi.org/10.1007/s00117-005-1306-y

Posted at the Zurich Open Repository and Archive, University of Zurich ZORA URL: https://doi.org/10.5167/uzh-156360

Journal Article

Published Version

Originally published at:

Zanetti, M; Saupe, N (2006). Magnetresonanztomographie der Schulter. Der Radiologe, 46(1):79-92.

DOI: https://doi.org/10.1007/s00117-005-1306-y 
Radiologe 2006 · 46:79-91 DOI 10.1007/s00117-005-1306-y

Online publiziert: 17. November 2005

(c) Springer Medizin Verlag 2005

\section{Rubrikherausgeber}

S. Delorme, Heidelberg

F. Kainberger, Wien

P. Reimer, Karlsruhe

W. Reith, Homburg/Saar

M. Uhl, Freiburg

\section{CME.springer.de}

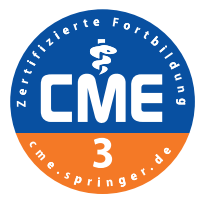

\section{Zertifizierte Fortbildung für} Kliniker und Niedergelassene!

Die CME-Teilnahme an diesem Fortbildungsbeitrag erfolgt online auf CME.springer.de und ist Bestandteil des Individualabonnements dieser Zeitschrift. Abonnenten können somit ohne zusätzliche Kosten teilnehmen.

Unabhängig von einem Zeitschriftenabonnement ermöglichen Ihnen CME.Tickets die Teilnahme an allen CME-Beiträgen auf CME.springer.de. Weitere Informationen zu CME.Tickets finden Sie auf CME.springer.de.

\section{Registrierung/Anmeldung}

Haben Sie sich bereits mit Ihrer Abonnementnummer bei CME.springer.de registriert? Dann genügt zur Anmeldung und Teilnahme die Angabe Ihrer persönlichen Zugangsdaten. Zur erstmaligen Registrierung folgen Sie bitte den Hinweisen auf CME.springer.de.

Online teilnehmen und 3 CME-Punkte sammeln

Die CME-Teilnahme ist nur online möglich. Nach erfolgreicher Beantwortung von mindestens 7 der 10 CME-Fragen senden wir Ihnen umgehend eine Bestätigung der Teilnahme und der $3 \mathrm{CME}$ Punkte per E-Mail zu.

\section{Zertifizierte Qualität}

Diese Fortbildungseinheit ist zertifiziert von der Landesärztekammer Hessen und der Nordrheinischen Akademie für Ärztliche Fort- und Weiterbildung und damit auch für andere Ärztekammern anerkennungsfähig.

Folgende Maßnahmen dienen der Qualitätssicherung aller Fortbildungseinheiten auf CME.springer.de: Langfristige Themenplanung durch erfahrene Herausgeber, renommierte Autoren, unabhängiger Begutachtungsprozess, Erstellung der CME-Fragen nach Vorgaben des IMPP mit Vorabtestung durch ein 22-köpfiges Board von Fachärzten.

Für Fragen und Anmerkungen stehen wir Ihnen jederzeit zur Verfügung:

Springer Medizin Verlag GmbH

Fachzeitschriften Medizin/Psychologie

CME-Helpdesk, Tiergartenstraße 17

69121 Heidelberg

E-Mail: cme@springer.com

CME.springer.de
M. Zanetti ${ }^{1} \cdot$ N. Saupe ${ }^{1,2}$

${ }^{1}$ Radiologie, Universitätsklinik Balgrist, Zürich

${ }^{2}$ Toronto, Canada

\section{Magnetresonanztomographie der Schulter}

\section{Impingement- und instabilitätsassoziierte Veränderungen}

\section{Zusammenfassung}

Die MRT der Schulter spielt neben Anamnese und klinischer Untersuchung eine bedeutende Rolle bei der Diagnose und Differenzierung zahlreicher Schulterpathologien und der Abklärung chronischer Schulterschmerzen. Wichtige Indikationsgebiete der MRT sind die Evaluation der Rotatorenmanschette und des Labrum-Kapsel-Apparats. Besondere Bedeutung hat die Beurteilung der Rotatorenmanschettenmuskulatur. Die immer noch kontrovers diskutierte MR-Arthrographie zeigt ihr größtes Potenzial bei der präzisen Unterscheidung von kleinen Rotatorenmanschettenläsionen, Labrum-Kapsel-Läsionen und Läsionen der langen Bizepssehne. In dem Beitrag wird auf die häufigsten pathologischen Veränderungen des Schultergelenks eingegangen. Daneben wird gezeigt, wie die Befunde für Schulterorthopäden beschrieben und quantifiziert werden.

\section{Schlüsselwörter}

Schulter · Rotatorenmanschette · Glenohumerale Ligamente $\cdot$ Labrum glenoidale . Bizepssehne

\section{MR imaging of the shoulder. Impingement- and instability-related abnormalities}

\begin{abstract}
In addition to the case history and the clinical examination, MR imaging has an important role in the diagnosis and differential diagnosis of numerous shoulder abnormalities and in the investigation of chronic shoulder pain. Important indications for MR imaging are any conditions or symptoms making assessment of the rotator cuff and the labrocapsular complex necessary. Assessment of the rotator cuff muscles, in particular, is crucial. The value of MR arthrography, which is still controversial, is discussed. The greatest potential benefit of MR arthrography is the accurate evaluation of subtle rotator cuff abnormalities and shoulder instability-related lesions, and the assessment of pathologic conditions of the long biceps tendon. This paper describes the most common pathologic findings of the shoulder joint and describes how the relevant findings are reported and quantified for the orthopaedic shoulder surgeon.
\end{abstract}

\section{Keywords}

Shoulder · Rotator cuff · Glenohumeral ligaments · Labrocapsular complex ·

Biceps tendon 
Bei der MR-Untersuchung der Schulter kommen die native MR-Technik sowie die direkte und indirekte MRArthrographie in Betracht.

\section{- Gadoliniumhaltiges Kontrastmittel}

Die Kontrastmittelinjektionsmenge beträgt 10-12 ml und erhöht sich pro stattgehabter Schulterluxation um 2-3 ml

Die Infektionsrate bei der diagnostischen Arthrographie ist klein

Die Supraspinatussehne ist am häufigsten von Verletzungen und Veränderungen betroffen.

\section{MR-Technik}

Grundsätzlich kommen bei der MR-Untersuchung der Schulter neben der nativen MRTechnik die direkte MR-Arthrographie mit Injektion eines gadoliniumhaltigen Kontrastmittels direkt ins Gelenk und die indirekte MR-Arthrographie mit i. v. Injektion eines gadoliniumhaltigen Kontrastmittels in Betracht. Bezüglich der Indikation der unterschiedlichen MR-Techniken existieren verschiedene Meinungen.

Der direkten MR-Arthrographie wird bei der Abklärung von Labrum, Knorpel, Kapsel- und Ligamentveränderungen der Vorzug gegeben. Im Übrigen ist sie bei der Differenzierung zwischen partieller und kleiner vollständiger Rotatorenmanschettenruptur der nativen MRT überlegen. Bei der MR-Arthrographie wird das $\mathbf{g a d o l i n i u m h a l t i g e ~ K o n - ~}$ trastmittel (Konzentration: $2 \mathrm{mMol} / \mathrm{ml}=1: 250$ verdünnt zur i. v. Konzentration) direkt ins Gelenk injiziert [18]. Die korrekte Nadelspitzenlage wird nach Injektion einer geringen Menge jodhaltigen Kontrastmittels in das Gelenk mittels fluoroskopischer Kontrolle geprüft. Als Injektionsweg eignet sich ein Zugang von ventral durch das Rotatorenmanschettenintervall. Der Zielort der Injektion projiziert sich in der Fluoroskopie auf das kraniale Drittel des glenohumeralen Gelenks 2-3 mm lateral des Gelenkspalts. Die Injektionsmenge beträgt 10-12 ml. Größere Volumina sollten nur nach rezidivierenden Schulterluxationen injiziert werden. Als Grundregel gilt, dass sich die Injektionsmenge um 2$3 \mathrm{ml}$ pro stattgehabte Schulterluxation erhöht [27]. Die Injektion des intraartikulären Kontrastmittels wird von den Patienten im Vergleich zum Liegen im MR-Scanner als weniger unangenehm empfunden [1]. Die Infektionsrate bei der diagnostischen Arthrographie ist klein. Eine Sammelstatistik aus dem Jahr 1975 zur Arthrographie ergab eine Infektionsrate von 3:126.0oo Untersuchungen [15].

Bei der indirekten MR-Arthrographie wird nach der i. v. Gadoliniuminjektion die Schulter durch den Patienten etwa 10-15 min bewegt [22].

Die Schulter wird in 3 Ebenen abgebildet:

- Die axiale Ebene umfasst kranial das gesamte Akromioklavikulargelenk

(AC-Gelenk) und distal den kaudalen Glenoidrand.

- Die parakoronale Ebene verläuft parallel zur Supraspinatussehne bzw. senkrecht zur Glenoidgelenkfläche.

- Die parasagittale Ebene verläuft senkrecht zur parakoronalen Ebene unter Einbezug der Sehnenansätze am Humeruskopf und der Rotatorenmanschettenmuskulatur.

Die Schichtdicken variieren zwischen 3 und $4 \mathrm{~mm}$. Bevorzugt werden Turbospinechosequenzen angewendet. Für die Labrumdiagnostik kommen auch Gradientenechosequenzen zur Anwendung.

Die MR-Protokolle sind abhängig von den Fragestellungen der Zuweiser, den Präferenzen des Radiologen, den technischen Möglichkeiten des MR-Scanners und den Entwicklungen der MR-Technologie. Unsere aktuellen Standardprotokolle sind in $\bullet$ Tabelle 1 zusammengefasst.

\section{Rotatorenmanschette}

Sie setzt sich von anterior nach posterior aus Subskapularis-, Supraspinatus-, Infraspinatus- und Teres-minor-Sehnen zusammen (• Abb. 1). Am häufigsten von Veränderungen und Verletzungen betroffen ist die Supraspinatussehne. Traumatische Rotatorenmanschettenveränderungen finden sich häufiger anterior-superior, während superior-posteriore Läsionen eher Ausdruck degenerativer Veränderungen sind [16].

Generell wird zwischen Tendinopathie, Partialruptur und transtendinöser Ruptur unterschieden. Tendinopathien sind gekennzeichnet durch Signalintensitätserhöhung auf Sequenzen mit kurzen Echozeiten (T1-gewichtet, protonengewichtet), aber fehlender Signalanhebung auf T2-gewichteten Sequenzen. Sie müssen von $>$ Magic-angle-Artefakten unterschieden werden [7]. Diese sind charakterisiert durch erhöhtes Signal in geordneten anatomischen Strukturen wie Sehnen und Ligamenten, die einen anatomischen 

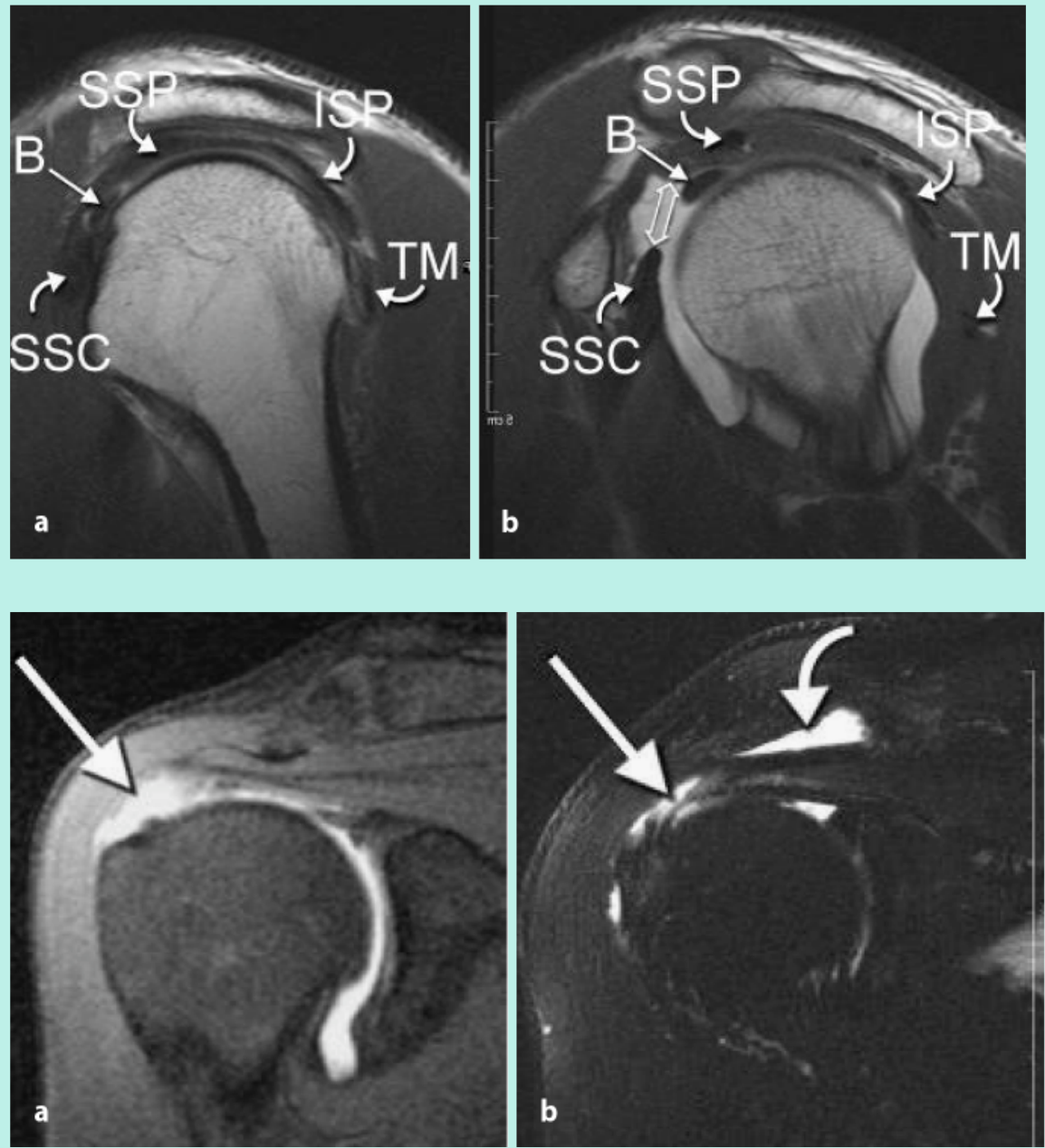

Abb. $2 \Delta$ Partialrupturen der Rotatorenmanschette, a T1-, b fettsaturierte, T2-gewichtete, parakoronale MR-Arthrographie-Sequenzen, Partialrupturen der Supraspinatussehne, definitionsgemäß nicht den gesamten Sehnenquerschnitt betreffend, Läsion entweder auf artikulärer ( $a$, Pfeil) oder auf bursaler Seite (b), b deutlich erkennbare Flüssigkeitsansammlung im Defekt (Pfeil) und in der Bursa subdeltoidea (gebogener Pfeil), c normale Supraspinatussehne mit glatter Begrenzung, Sehnenunterfläche (Pfeilspitzen) in T1-gewichteter fettunterdrückter MR-Arthrographie-Aufnahme

Verlauf von $55^{\circ}$ gegenüber dem Hauptmagnetfeld einnehmen. Magic-angle-Artefakte kommen nur auf Sequenzen mit kurzen Echozeiten $(<37 \mathrm{~ms})$ vor.

Für $\gg$ Partialrupturen $(\bullet$ Abb. 2) der Supra- und Infraspinatussehnen ist typisch, dass sie nicht den gesamten Querschnitt der Sehne betreffen. Die Läsion kann entweder auf der artikulären oder auf der bursalen Seite manifestiert sein.

Bei der $\$ transtendinösen Ruptur sind die pathologischen Veränderungen im gesamten Sehnenquerschnitt abgrenzbar (• Abb. 3).

Die \Subskapularissehnenrupturen werden anders klassifiziert. Neben Oberrandläsionen mit Befall des kranialen Drittels lassen sich Partialrupturen mit Befall der kranialen Hälfte der Sehneninsertion und komplette Rupturen mit vollständigem Abriss der gesamten Sehneninsertion abgrenzen [17].

Rotatorenmanschettenrupturen können zu Atrophie und Verfettung der Rotatorenmanschettenmuskulatur führen. Tierexperimentell wurde dies schon nach 4 Wochen beobachtet [3]. Klinisch spielt dies bei der Wahl der Operationstechnik eine wichtige Rolle [9]. Bei fortgeschrittener Atrophie und Verfettung der Muskulatur kommen rekonstruktive Verfahren zur Rückgewinnung der Funktionalität nicht mehr in Frage. Entsprechend spielt die Beurteilung der Rotatorenmanschettenmuskulatur eine wichtige Rolle.
Abb. $1 \varangle$ Rotatorenmanschettensehnen, parasagittale T1-gewichtete MR-Arthrographie der Schulter, normale Anatomie der Rotatorenmanschette in 2 konsekutiven Schichten (a peripherer als $\mathrm{b}$ ), von anterior nach posterior aus Subskapularis- (SSC), Supraspinatus- (SSP), Infraspinatus- (ISP) und Teres-minor-Sehnen (TM) (gebogene Pfeile) bestehend; B, gestreckter Pfeil lange Bizepssehne, offener Doppelpfeil Rotatorenmanschettenintervall zwischen Supraspinatus- und Subskapularissehne

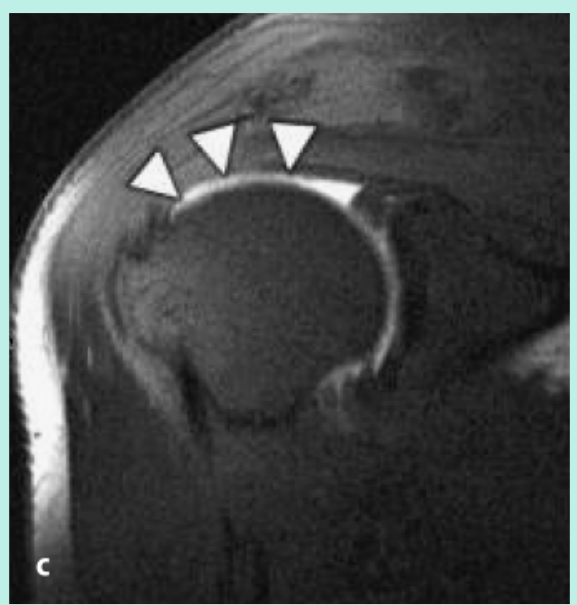

\section{- Partialruptur}

\section{Transtendinöse Ruptur}

\section{Subskapularissehnenruptur}

Bei fortgeschrittener Atrophie und Verfettung der Rotatorenmanschettenmuskulatur sind rekonstruktive Verfahren kontraindiziert 


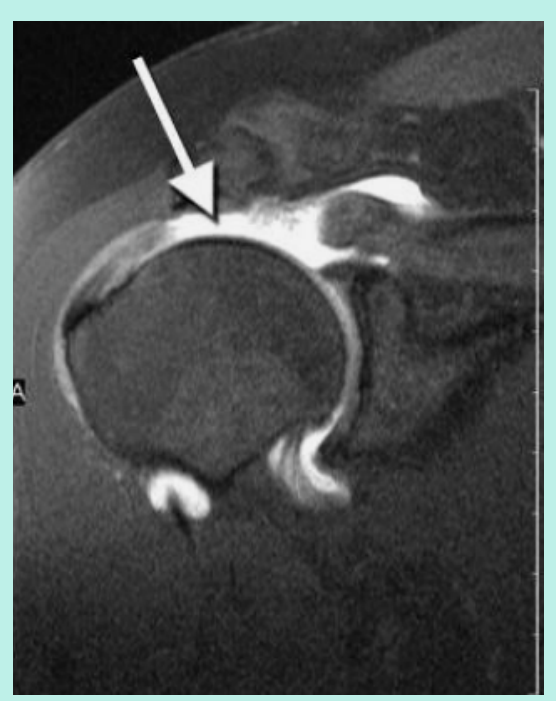
spinatussehne, T1-gewichtete fettsaturierte MR-Arthrographie, abgrenzbare pathologische Veränderungen im gesamten Sehnenquerschnitt (Pfeil), retrahierte Supraspinatussehne
Abb. $3 \Delta$ Transtendinöse Ruptur der Supra-

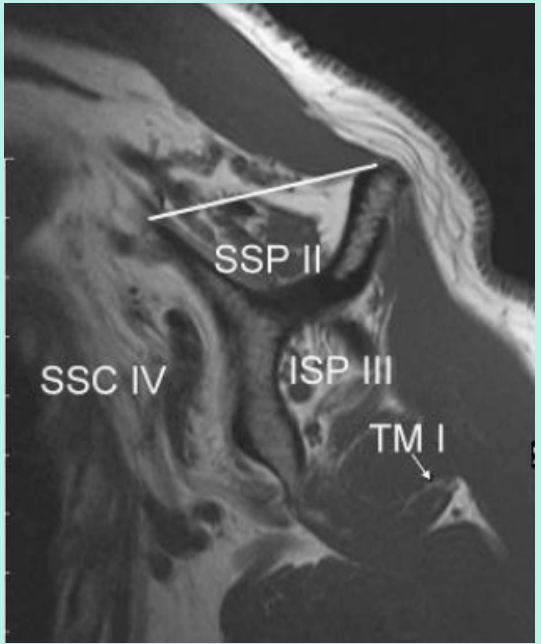

Abb. $4 \Delta$ Verfettung und Atrophie der Rotatorenmanschettenmuskulatur, T1-gewichtete parasagittale Sequenz in der Fossa supraspinata, unterschiedliche Verfettungsgrade der Rotatorenmanschettenmuskulatur: nach Goutallier-Klassifikation Grad-I-Verfettung des $M$. teres minor (TM I) mit nur vereinzelten Fettstreifen (Pfei), Grad-II-Verfettung des M. supraspinatus (SSP II) mit weniger Fett- als Muskelmasse, Grad-III-Verfettung des M. infraspinatus (ISP III) mit in etwa gleicher Fettund Muskelmasse, Grad-IV-Verfettung des M. subscapularis (SSCIV) mit mehr Fett- als Muskelmasse, Atrophie des M. supraspinatus mit positiven Tangentenzeichen: nicht atrophierter Muskelbauch des M. supraspinatus sollte Tangente über den kranialen Begrenzungen der Spina scapulae und des Korakoids kreuzen
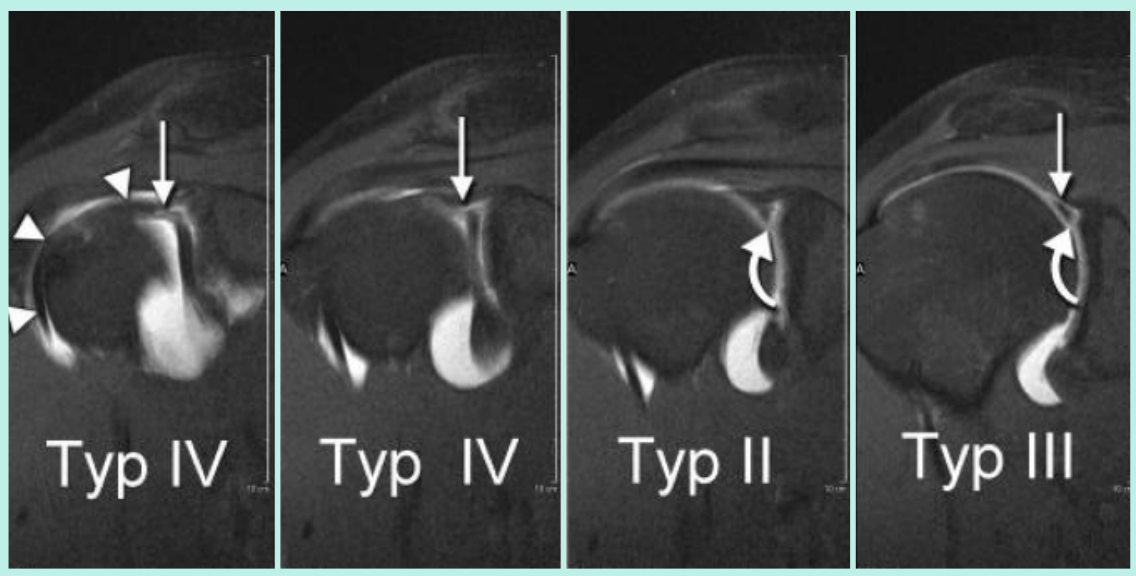

Abb. $6<$ SLAP-Läsionen, parakoronale T1-gewichtete fettsaturierte MR-Arthrographie-Aufnahmen; Schwierigkeit der genauen Typisierung: Typisierung beim gleichen Patienten von anterior nach posterior (von links nach rechts) deutlich verändert: ganz anterior (linke Bildreihe) Typ-IV-SLAP-Läsion mit Rissausläufer nicht nur bis zur Insertion der langen Bizepssehne, sondern mit zusätzlichem Bizepssehnenlängsriss (Pfeil); Typ-II-Läsion, charakterisiert durch bis zur Bizepssehneninsertion verlaufenden Riss (gebogener Pfeil) im kranialen Labrum; ganz rechts Typ-III-SLAP-Läsion mit korbhenkelähnlichem Riss (gerader und gebogener Pfeil)

\section{- Goutallier-Klassifikation}

Ihre Verfettung kann mit der $\triangleright$ Goutallier-Klassifikation eingeschätzt werden ( $\bullet$ Tabelle 2) (• Abb. 4). Diese basiert initial auf einer CT-Studie [9], die später mittels MRT validiert wurde [8]. Daneben wird für die Beurteilung der Atrophie der Supraspinatusmuskulatur auch das Tangentenzeichen verwendet [31]. Die normale, nicht atrophierte Supraspinatusmuskulatur kreuzt eine Tangente, welche auf parasagittalen MR-Aufnahmen zwischen dem Oberrand der Spina scapulae und dem des Korakoids gezogen wird. Bleibt die Rotatorenmanschettenmuskulatur unterhalb der oben beschriebenen Tangente, liegt ei- 
Tabelle 1

\section{MR-Protokolle}

\begin{tabular}{|llrllll}
\hline Sequenz & Ebene & $\begin{array}{l}\text { TR } \\
{[\mathrm{ms}]}\end{array}$ & $\begin{array}{l}\text { TE } \\
{[\mathrm{ms}]}\end{array}$ & $\begin{array}{l}\text { Schicht- } \\
\text { dicke } \\
{[\mathrm{mm}]}\end{array}$ & $\begin{array}{l}\text { FOV } \\
{[\mathrm{mm}]}\end{array}$ & Matrix \\
\hline Nativ-MR-Protokoll (v. a. postoperativ) & & & & & \\
\hline Arthro-MR-Protokoll & & & & & & \\
\hline pd_tse_fs & Parakoronal & 2870 & 13 & 4 & $160 \times 100$ & 512 \\
\hline t2_tse_fs & Parakoronal & 3460 & 92 & 4 & $160 \times 100$ & 512 \\
\hline t1_tse_fs & Parakoronal & 648 & 12 & 3 & $160 \times 100$ & 512 \\
\hline t1_se & Parasagittal & 450 & 12 & 4 & $160 \times 100$ & 512 \\
\hline t1_se & Axial & 450 & 12 & 3 & $160 \times 100$ & 512 \\
\hline pd_tse_fs & Parakoronal & 2870 & 13 & 4 & $160 \times 100$ & 512 \\
\hline STIR & Parakoronal & 4800 & 26 & 3 & $160 \times 100$ & 512 \\
\hline t1_se & Parasagittal & 450 & 12 & 4 & $160 \times 100$ & 512 \\
\hline STIR & Parasagittal & 4930 & 29 & 4 & $160 \times 100$ & 512 \\
\hline pd_tse_fs & Axial & 2870 & 13 & 4 & $160 \times 100$ & 512 \\
\hline
\end{tabular}

pd_tse_fs protonengewichtete Turbospinechosequenz mit Fettsaturierung, $\mathrm{t} 1$ (t2)_tse_fs $T 1$ (T2)gewichtete Turbospinechosequenz mit Fettsaturierung, STIR Short-inversion-time-inversion-recoverySequenz
Tabelle2

Graduierung der Rotatorenman-

schettenmuskulaturverfettung

nach Goutallier et al. [9]

\begin{tabular}{ll} 
Grad I & $\begin{array}{l}\text { Vereinzelte Fettstreifen in der } \\
\text { Muskulatur }\end{array}$ \\
\hline Grad II & Fettmasse<Muskelmasse \\
\hline Grad III & Fettmasse=Muskelmasse \\
\hline Grad IV & Fettmasse>Muskelmasse
\end{tabular}

Tabelle 3

SLAP-Läsionen-Klassifikation

nach Snyder et al. [19]

\begin{tabular}{|cl|}
\hline Typ I & $\begin{array}{l}\text { Gefranste Oberfläche des } \\
\text { Labrums oder/und kleine } \\
\text { Einrisse }\end{array}$ \\
\hline Typ II & $\begin{array}{l}\text { Riss im kranialen Labrum bis } \\
\text { zur Bizepssehneninsertion hin }\end{array}$ \\
\hline Typ III & $\begin{array}{l}\text { Korbhenkelrissähnlicher } \\
\text { Labrumriss }\end{array}$ \\
\hline Typ IV & $\begin{array}{l}\text { Wie Typ III mit einem zusätzli- } \\
\text { chen Bizepssehnenlängsriss }\end{array}$ \\
\hline
\end{tabular}

ne substanzielle Atrophie vor (mehr als 2 Standardabweichungen unter der Altersnorm), das Tangentenzeichen ist positiv.

Der Ultraschall erlaubt im Gegensatz zur MRT und CT nur eine grobe Differenzierung zwischen normaler Muskulatur, substanzieller Atrophie und fettiger Degeneration [21]. Zudem beschränkt sich die sonographische Beurteilung auf die Supraspinatus-, Infraspinatus- und Teres-minor-Muskulatur.

Die Beurteilung der Rissgröße spielt hinsichtlich der Entscheidung, ob offen oder arthroskopisch revidiert werden sollte, heute nur noch eine untergeordnete Rolle, da auch große Rupturen $(>4 \mathrm{~cm}$ ) primär arthroskopisch angegangen werden.

\section{AC-Gelenk}

Seine Beurteilung steht im engen Zusammenhang mit pathologischen Veränderungen der Rotatorenmanschette. Bei der AC-Gelenkarthrose sollten Osteophyten mit kaudaler Ausdehnung und die Gelenkkapselhypertrophie beschrieben werden. Eine Gelenkkapselverdickung $>3 \mathrm{~mm}$ sowie subkortikale ödemartige Knochenmarkalterationen sind Hinweise für das Vorliegen eines schmerzhaften AC-Gelenks [20].

Die Beschreibung des $>$ Os acromiale, welches einen nicht fusionierten Ossifikationskern darstellt, ist für den Schulterorthopäden wichtig. Es ist in 4 von 5 Fällen instabil und wird bei Rotatorenmanschettenoperationen häufig gleichzeitig stabilisiert. Es kommt in der Gesamtbevölkerung in 6-8\% vor. Die Beschreibung eines Os acromiale auf MR-Bildern ist bedeutsam, da es auf Standardröntgenaufnahmen übersehen werden kann. Am besten lässt es sich auf axialen MR-Bildern erkennen.

\section{Bursa subacromiale/Bursa subdeltoidea}

Sie kommunizieren miteinander. Flüssigkeitsansammlungen in ihnen sind häufig mit Rotatorenmanschettenläsionen assoziiert, insbesondere bei transtendinösen Rupturen. Eine vermehrte Flüssigkeitsansammlung in diesen Bursen ohne das Vorliegen von Rotatorenmanschettenrupturen kann Ausdruck einer Bursitis sein. Häufiger sind die Flüssig-
Eine Gelenkkapselverdickung $>3 \mathrm{~mm}$ sowie subkortikale ödemartige Knochenmarkalterationen weisen auf ein schmerzhaftes AC-Gelenk hin

\section{- Os acromiale}


Eine Tendinopathie der langen Bizepssehne ist durch Signalalteration auf Sequenzen mit kurzen Echozeiten zu diagnostizieren

\section{- Kaliberschwankung}

Bizepssehnenrupturen werden bevorzugt auf parasagittalen Aufnahmen diagnostiziert

\section{SLAP-Läsion}

Ein Läsionsausläufer nach medial zum Glenoid hin entspricht eher einem physiologischen Sulcus, ein Rissausläufer nach lateral zur Bizepssehne hin eher einer SLAP-Läsion keitsansammlungen Ausdruck einer anderen Schulterpathologie, wie eines Rotatorenmanschettenimpingements mit Tendinopathie der Supraspinatussehne oder einer Labrumläsion [12]. Geringe Flüssigkeitsansammlungen können auch bei asymptomatischen Personen nachgewiesen werden. Signalintensitätserhöhung auf flüssigkeitsempfindlichen Sequenzen, hinweisend auf eine Bursitis, sind in der postoperativen Schulter Ausdruck normaler postoperativer Veränderungen und sollten nicht fälschlicherweise als Bursitis interpretiert werden [33].

\section{Lange Bizepssehne und Bizepsanker}

Läsionen der langen Bizepssehne können isoliert, jedoch häufiger im Zusammenhang mit Rotatorenmanschettenläsionen auftreten. Insbesondere bei Rupturen der Supra- und Infraspinatussehne mit nachfolgendem Schulterkopfhochstand kann es zu einer Irritation der langen Bizepssehne kommen. Diese kann sowohl eine Tendinopathie, eine Partialruptur oder eine vollständige Ruptur aufweisen. Am häufigsten sind Läsionen der langen Bizepssehne im Rotatorenmanschettenintervall anzutreffen. Eine Tendinopathie ist wie bei der Rotatorenmanschettenruptur durch Signalalteration auf Sequenzen mit kurzen Echozeiten zu diagnostizieren, wobei jedoch neben dem Magic-angle-Artefakt das Partialvolumenproblem die Diagnose deutlich erschwert. Partialrupturen gehen mit -Kaliberschwankungen (umschriebene Verdickung, umschriebene Ausdünnung) der Sehne einher (•Abb.5).

Die komplette lange Bizepssehnenruptur ist gekennzeichnet durch fehlende Abgrenzung im Rotatorenmanschettenintervall. Bizepssehnenrupturen werden bevorzugt auf parasagittalen Aufnahmen diagnostiziert [32]. Häufig finden sich im Sulcus intertubercularis auf axialen Aufnahmen eine Restsehnenstruktur bzw. einzelne fibrotische Restfasern. Diese sollten nicht fälschlicherweise als intakte oder nur leicht lädierte lange Bizepssehne interpretiert werden.

Am Bizepsanker werden die \SLAP-Läsionen differenziert. SLAP ist definiert als eine Läsion des superioren Labrums mit a.-p. Ausdehnung. Die Erstbeschreibung von Snyder et al. [19] basierte auf 4 Typen (- Abb. 6) (•Tabelle 3). Die Klassifikation wurde in der Zwischenzeit durch Zusatztypen ergänzt, die die Ausdehnung der Läsion in die umliegenden Strukturen beschreiben. Diese Zusatztypisierung hat sich jedoch wegen ihrer Komplexizität bei Schulterchirurgen nicht durchsetzen können. Die SLAP-Läsionen Typ I und III sind stabil, Typ II und IV sind instabil. Die SLAP-Diagnose wird erschwert durch die schwierige Differenzierung von einem physiologisch auftretenden Sulcus zwischen superiorem Labrum und hyalinem Knorpel des Glenoids. Als Grundsatzregel gilt, dass ein Läsionsausläufer nach medial zum Glenoid hin eher einem physiologischen Sulcus, ein Rissausläufer nach lateral zur Bizepssehne hin eher einer SLAP-Läsion entspricht. Die in der Literatur angegebenen Treffsicherheiten zur Beurteilung der SLAP-Läsionen variieren stark [24].

\section{Glenohumerale Ligamente}

Es werden 3 glenohumerale Ligamente unterschieden:

- Das superiore glenohumerale Ligament erkennt man auf den axialen Aufnahmen auf Höhe des Processus coracoideus, wo es vom Glenoid parallel zum Processus coracoideus zum Humeruskopf hin verfolgbar ist.

- Das superiore glenohumerale Ligament geht kaudal in das mittlere glenohumerale Ligament über.

- Das inferiore glenohumerale Ligament verläuft vom inferioren Glenoidrand zur kaudalen Gelenkkapsel (• Abb. 7).

Das superiore glenohumerale Ligament ist bei der $>$ Poulie-Läsion betroffen [23]. Das Poulie oder Pulley (frz. bzw. engl. für Umlenkrolle) ist eine bindegewebige Struktur, welche als Schlinge verhindert, dass die lange Bizepssehne im Rotatorenmanschettenintervall nach medial subluxiert. Es setzt sich aus Anteilen des superioren glenohumeralen Ligaments, des Lig. coracohumerale und des Subskapularissehnenoberrands zusammen. 

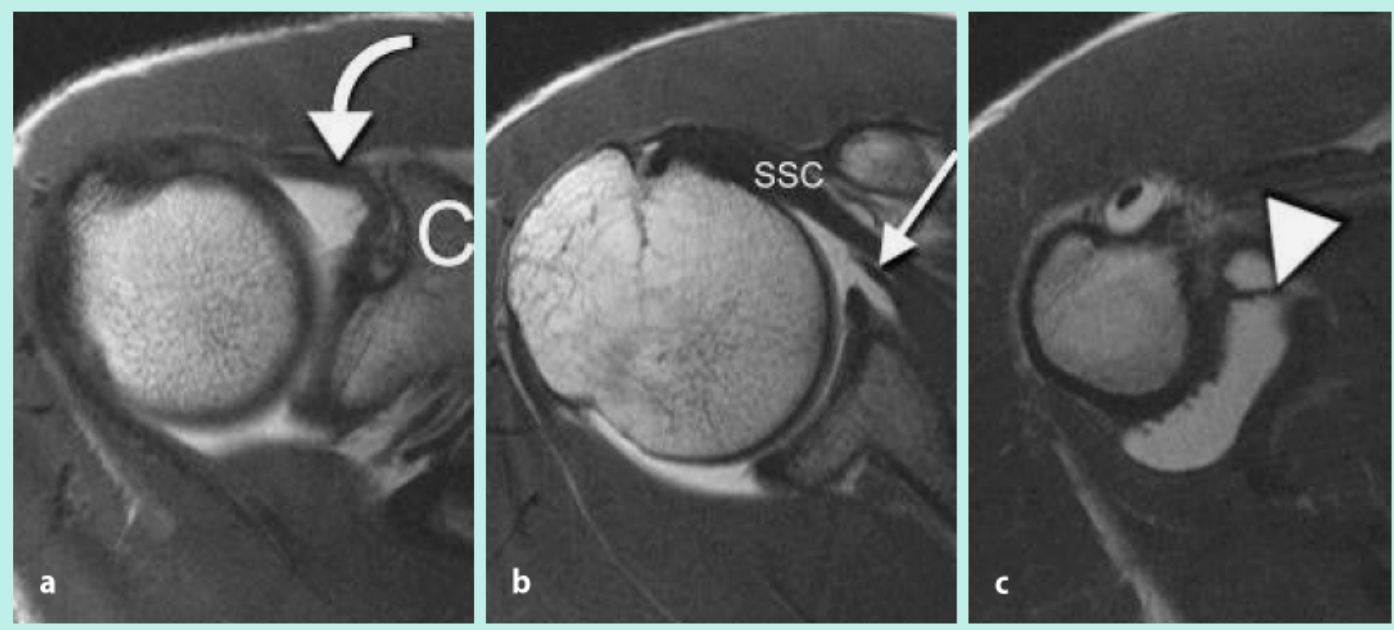

Abb. $7 \Delta$ Glenohumerale Ligamente, axiale T1-gewichtete MR-Arthrographie-Bilder, a normales superiores glenohumerales Ligament (gebogener Pfeil) mit Ursprung am Glenoidrand und parallel zum Korakoid $(C)$ zum Humeruskopf verlaufend, b superiores glenohumerales Ligament, kaudal ins mittlere glenohumerale Ligament (gerader Pfeil) übergehend, c inferiores glenohumerales Ligament (Pfeilspitz), vom inferioren Glenoidrand zur kaudalen Gelenkkapsel verlaufend, SSC Subskapularissehne

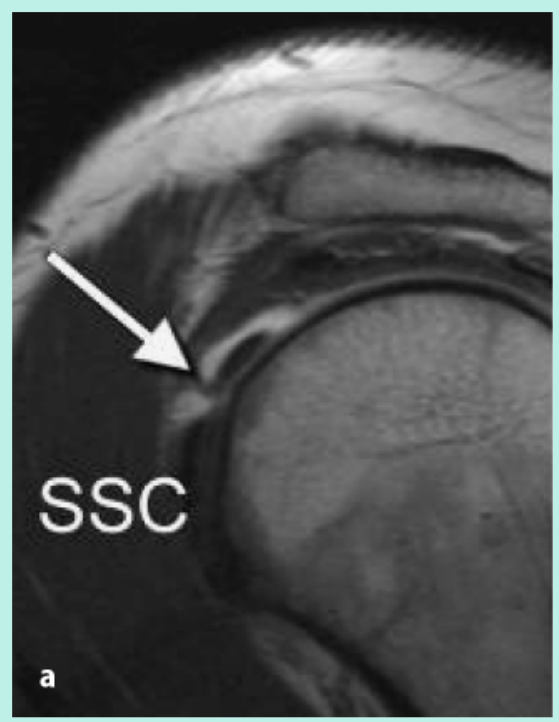

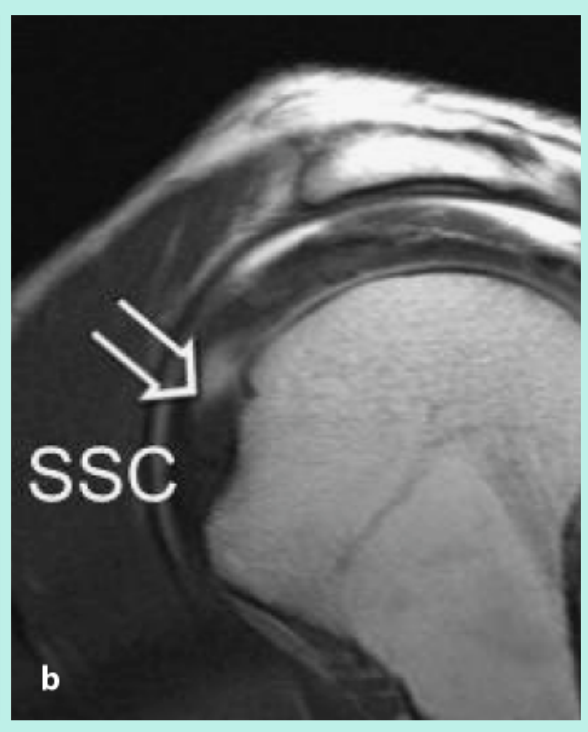

Abb. $8<$ Poulie (Zusammenfluss des superioren glenohumeralen Ligaments, des Lig. coracohumerale und des Subskapularissehnenoberrands), parasagittale T1-gewichtete MR-Aufnahmen, a normales Poulie (Pfeil), b lädiertes Poulie (offener Pfeil), charakteristische Signalintensitätserhöhungen im Subskapularissehnenoberrand (offener Pfeil)
Die Poulie-Läsion wird am zuverlässigsten auf parasagittalen Bildern diagnostiziert und ist charakterisiert durch Signalintensitätserhöhungen im Subskapularissehnenoberrand (- Abb. 8) [25]. Weitere Kriterien sind die mediale Subluxation der langen Bizepssehne, sichtbar auf axialen Aufnahmen und eine Kontrastmittelansammlung ventral der Subskapularissehne.

Läsionen der glenohumeralen Ligamente sind auch mit der MR-Arthrographie schwierig zu diagnostizieren [5]. Bei vorderer Schulterluxation ist das inferiore glenohumerale Ligament am häufigsten rupturiert. Ein Ausriss der glenohumeralen Ligamente an der humeralen Insertion wird als $>$ HAGL-Läsion („,humeral avulsion of the glenohumeral ligament") bezeichnet. Diese ist selten und manifestiert sich durch eine Vergrößerung des Recessus axillaris und eine Konturirregularität an der humeralen Insertion. Gelegentlich ist die Läsion mit einem zusätzlichen ossären Ausriss aus dem Humerus vergesellschaftet und wird dann als bony HAGL oder BHAGL bezeichnet [4] (• Abb. 9).

Kapsel- und Ligamentveränderungen spielen eine wichtige Rolle bei der Diagnose der adhäsiven Kapsulitis („frozen shoulder“). Die orthopädische Literatur [26] be-
Die Poulie-Läsion wird am zuverlässigsten auf parasagittalen Bildern diagnostiziert

\section{- HAGL-Läsion}

\section{Adhäsive Kapsulitis}



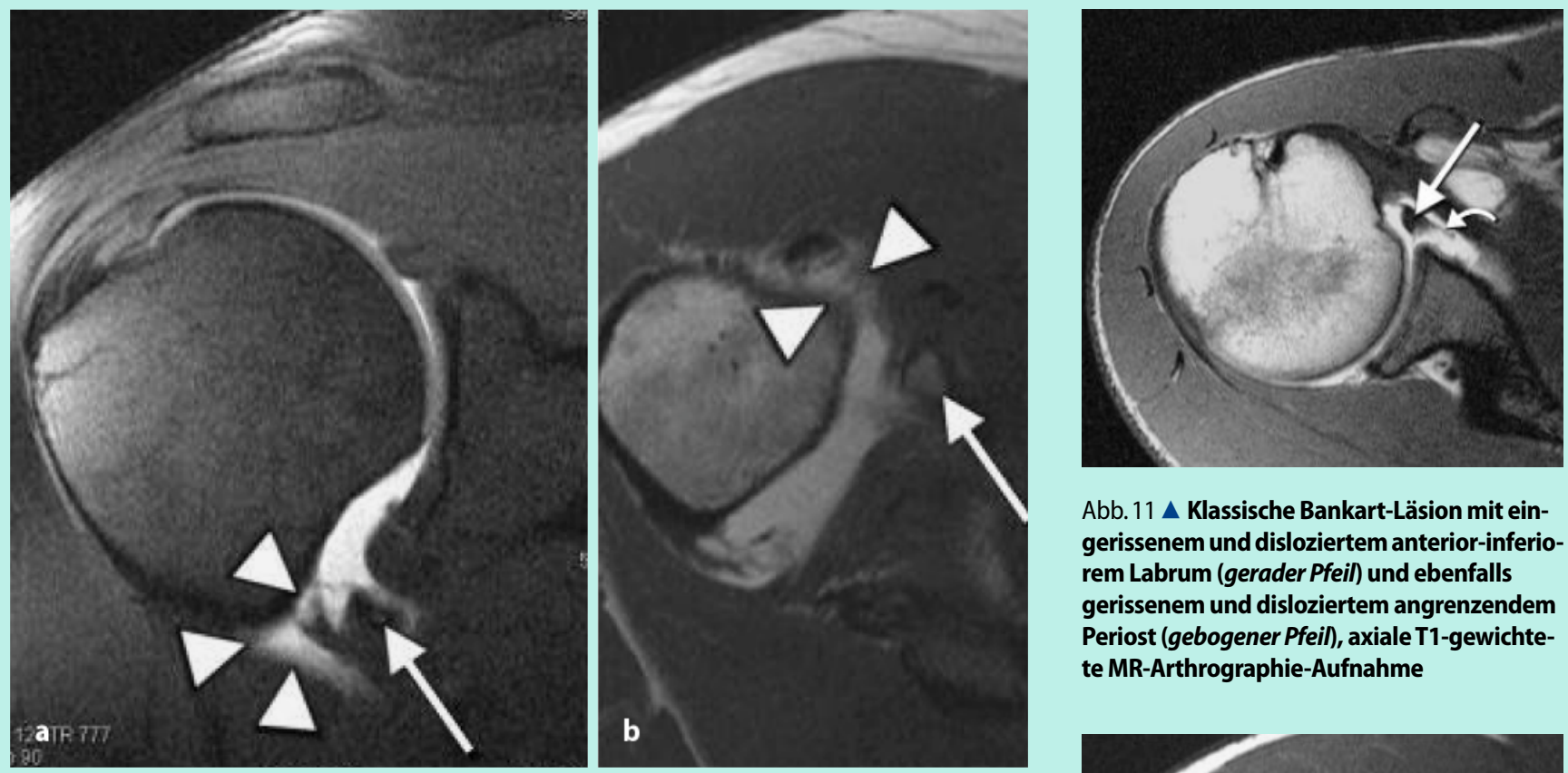

Abb. $11 \Delta$ Klassische Bankart-Läsion mit eingerissenem und disloziertem anterior-inferiorem Labrum (gerader Pfeil) und ebenfalls gerissenem und disloziertem angrenzendem Periost (gebogener Pfeil), axiale T1-gewichtete MR-Arthrographie-Aufnahme

Abb. $9 \Delta$ HAGL-Läsion, a parakoronale T1-gewichtete fettsaturierte, b 11-gewichtete nicht fettsaturierte MR-Arthrographie-Aufnahme, Vergrößerung des Recessus axillaris, Konturirregularität an der humeralen Insertion des inferioren glenohumeralen Ligaments (Pfeilspitzen), zusätzlicher ossärer Ausriss (Pfeil) aus dem Humerus: bony HAGL oder BHAGL
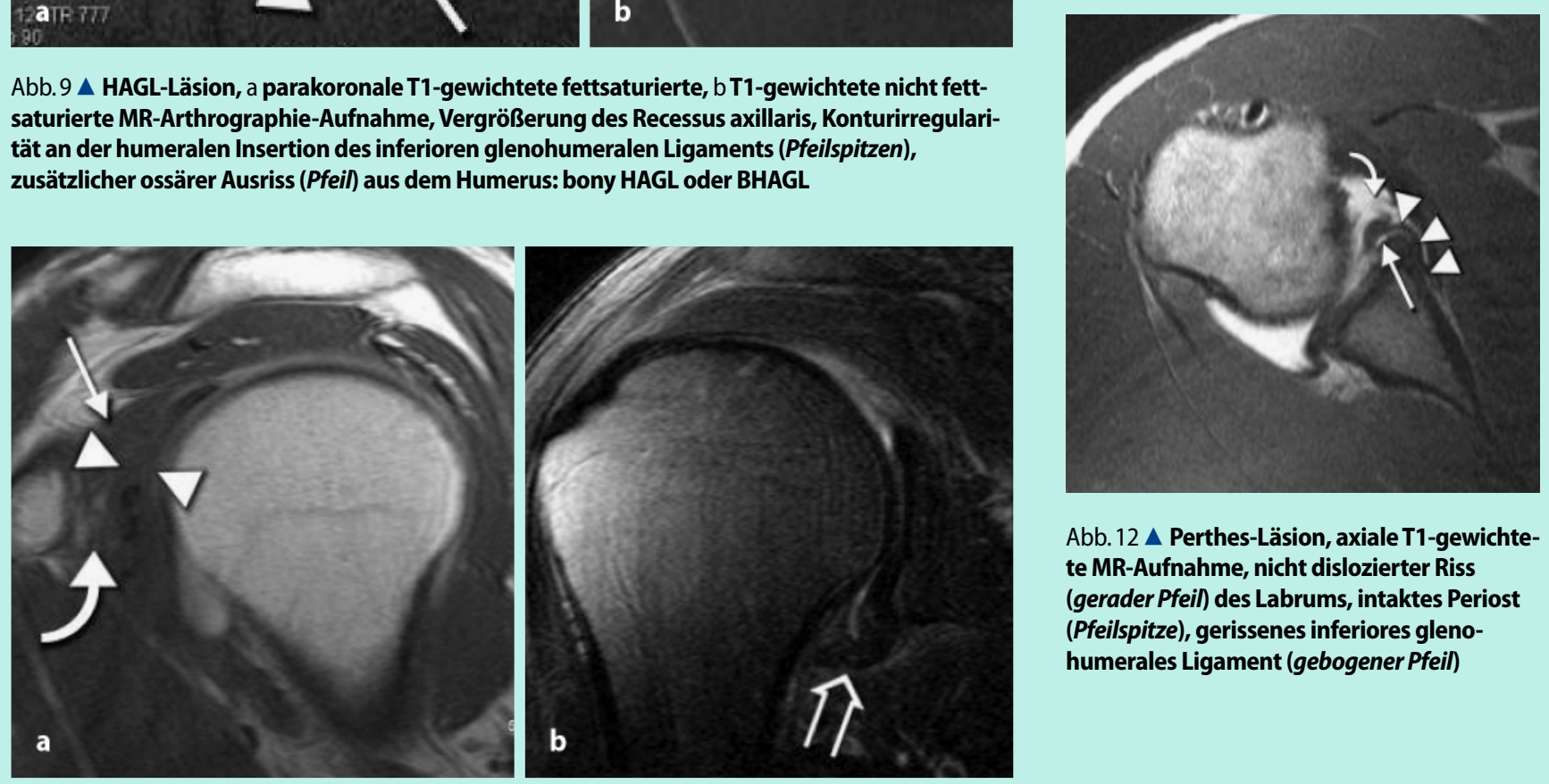

Abb. $12 \Delta$ Perthes-Läsion, axiale T1-gewichtete MR-Aufnahme, nicht dislozierter Riss (gerader Pfeil) des Labrums, intaktes Periost (Pfeilspitze), gerissenes inferiores glenohumerales Ligament (gebogener Pfeil)

Abb. $10 \Delta$ Adhäsive Kapsulitis, a T1-gewichtete parasagittale, b T2-gewichtete parakoronale fettsaturierte MR-Arthrographie-Aufnahme, a Verdickung der Gelenkkapsel (Pfeilspitzen) (dicker als $6 \mathrm{~mm}$ ) auf Höhe des Rotatorenmanschettenintervalls, Verdickung des korakohumeralen Ligaments (gerader Pfeil), Obliteration (gebogener Pfeil) des subkorakoidalen Fettgewebes als zusätzlicher Hinweis, b verklebter Recessus axillaris, Kapsel ebenfalls verdickt (offener Pfeil)

Hinweis für eine adhäsive Kapsulitis ist u. a. eine Gelenkkapseldicke $>6 \mathrm{~mm}$ auf Höhe des Rotatorenmanschettenintervalls tont, dass sie mit pathologischen Veränderungen im Rotatorenmanschettenintervall einhergeht, aber nicht, wie häufig in der radiologischen Literatur angegeben, mit pathologischen Veränderungen im Recessus axillaris [6]. Im Rotatorenmanschettenintervall finden sich bei adhäsiver Kapsulitis eine verdickte Kapsel und eine Synovitis. Entsprechend sollten pathologische Veränderungen in dieser Region gesucht werden. Sowohl eine Gelenkkapseldicke breiter als $6 \mathrm{~mm}$ auf Höhe des Rotatorenmanschettenintervalls als auch eine Verdickung (>3 mm) des korakohumeralen Ligaments sind Hinweise für eine adhäsive Kapsulitis. Ein zusätzliches Zeichen stellt die Obliteration des subkorakoidalen Fettgewebes dar (• Abb. 10). Normalerweise ist der subkorakoidale Raum mit normalem 


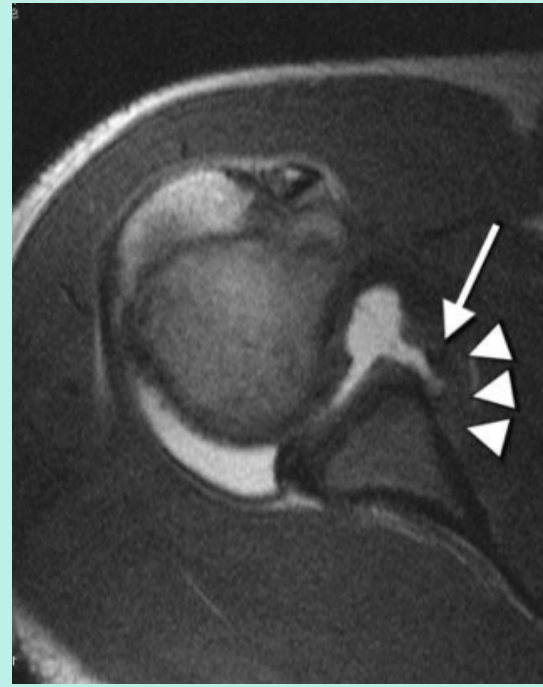

Abb. $13 \triangle$ ALPSA-Läsion, axiale T1-gewichtete MR-Aufnahme, disloziertes Labrum (gerader Pfeil), nach medial hin aufgewickeltes, verdickt erscheinendes Periost (Pfeilspitzen) ist dennoch intakt

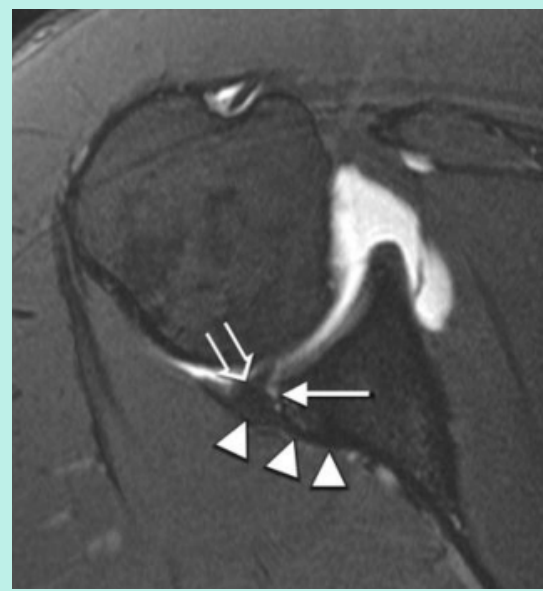

Abb. $14 \triangle$ POLPSA-Läsion, MR-Arthrographie, wenig disloziertes posteriores Labrum (offener Pfeil), intakte posteriore

Gelenkkapsel, abgelöstes posteriores noch am posterioren Glenoid befestigtes Periost (Pfeilspitzen), einen kleinen mit dem Gelenk kommunizierenden Recessus (gerader Pfeil) erzeugend
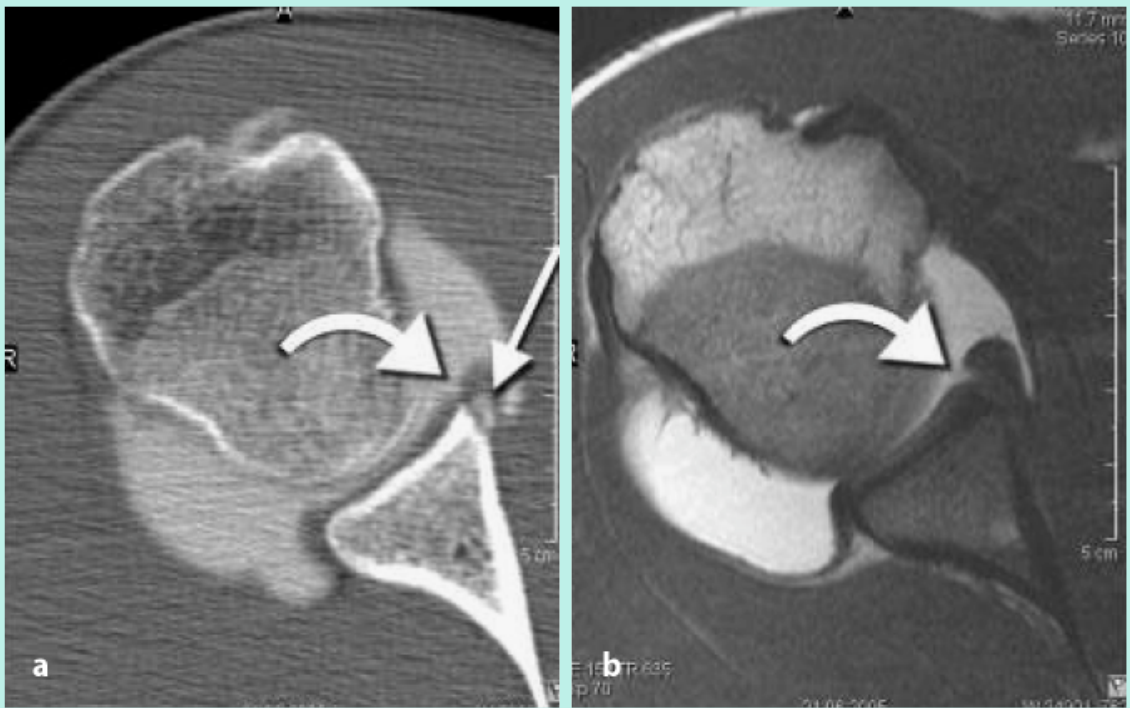

Abb. $15 \Delta$ Ossäre Glenoidrandläsion, a axiale Arthro-CT, $b$ axiale T1-gewichtete MRT, bessere Abgrenzung der ossären Glenoidrandverletzungen (gerader Pfeil) in Arthro-CT (a), gebogener Pfeil Labrumläsion
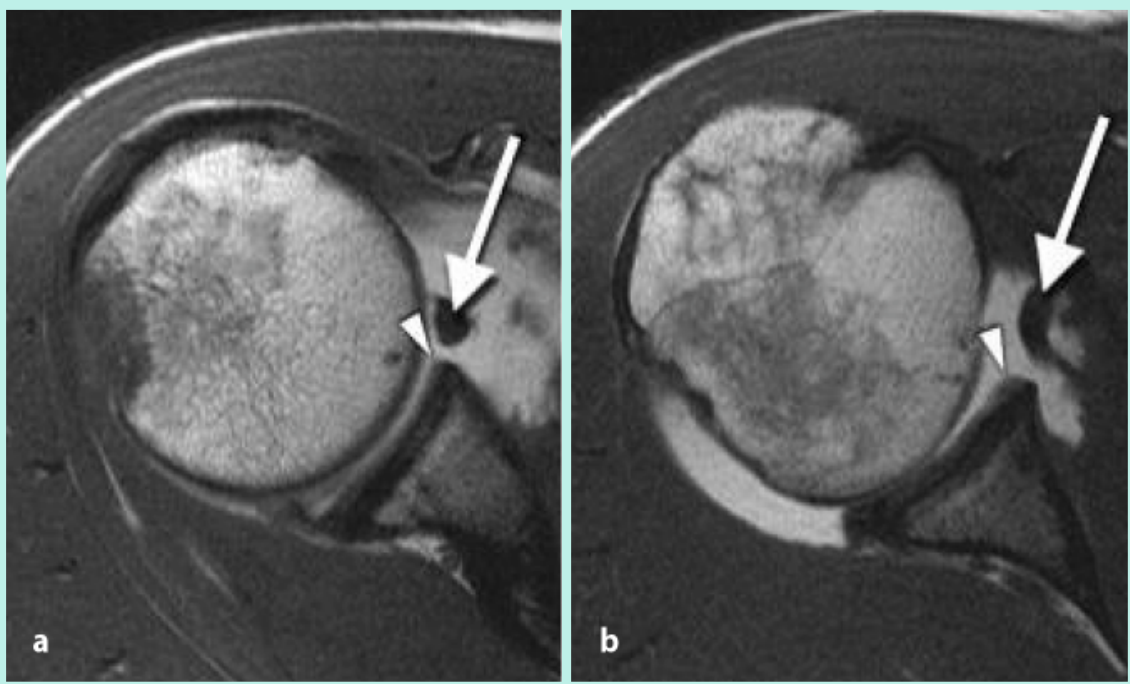

Abb. $16 \triangle$ Buford-Komplex, axiale T1-gewichtete MR-Aufnahme, fehlendes Labrum anteriorsuperior (Pfeilkopf), Verdickung des mittleren glenohumeralen Ligaments (Pfeil)

Fettgewebe ausgefüllt. Die Beurteilung der Gelenkkapsel im Recessus axillaris ist wahrscheinlich weniger zuverlässig als vormals beschrieben. Dessen Verkleinerung auf MRArthrographie-Bildern gilt dennoch als weiterer Hinweis für das Vorliegen einer „frozen shoulder" [11].

\section{Labrum glenoidale}

Es ist im Normalfall auf MR-Aufnahmen scharf begrenzt, dreiecksförmig und weist auf allen MR-Sequenzen ein hypointenses Signal auf. Labrumläsionen werden nach vorderer 
Bankart-Läsion

\section{- Perthes-Läsion}

Bei der ALPSA-Läsion sind Labrum und Periost vom Glenoid abgehoben, das Periost ist im Gegensatz zur Bankart-Läsion intakt

\section{- POLPSA-Läsion}

Ossäre Glenoidrandläsionen können in der Arthro-CT besser abgegrenzt werden als in der MRT

\section{- Buford-Komplex}

Anterior-inferiore Veränderungen sind häufig pathologischer Natur,

Bei Patienten mit einer Impingementsymptomatik beträgt die Prävalenz von glenohumeralen Knorpelläsionen $33 \%$ unterer Schulterluxation am häufigsten anterior-inferior angetroffen. Sie werden als Bankart-Läsion (• Abb. 11) klassifiziert, wenn eine Schädigung des anteriorinferioren Labrums mit einer Ablösung des ventralen Bands des inferioren glenohumeralen Ligaments und des angrenzenden Periosts assoziiert ist.

Eine Perthes-Läsion stellt eine nichtdislozierte Variante der Bankart-Läsion dar [29]. Das Periost bleibt intakt (- Abb. 12).

Bei der ALPSA-Läsion („anterior labrum ligament periosteal sleeve avulsion") sind das Labrum und das Periost vom Glenoid abgehoben, das Periost ist aber im Gegensatz zur Bankart-Läsion intakt (• Abb. 13) [13]. Das lädierte anterior-inferiore Labrum wird durch das ventrale Band des inferioren glenohumeralen Ligaments vom Glenoid weggezogen.

Ähnlich zur ALPSA-Läsion, welche das anterior-inferiore Labrum betrifft, beschreibt man dorsal eine $>$ POLPSA-Läsion („posterior labrocapsular periostal sleeve avulsion“). Diese zeigt ein disloziertes posteriores Labrum bei abgelöstem posteriorem Periost, welches jedoch wie bei der ALPSA nicht zerrissen und noch am posterioren Glenoid befestigt ist [30] (• Abb. 14).

Die therapeutische Relevanz der Klassifizierung in Bankart-, Perthes-, ALPSA- und POLPSA-Läsion ist wahrscheinlich kleiner als die Unterscheidung von Labrumläsion mit und ohne ossärer Glenoidrandläsion. Unglücklicherweise können ossäre Glenoidrandläsion in der MRT auch bei hoher Bildauflösung übersehen werden. Die Arthro-CT vermag sie treffsicherer abzugrenzen [2] (• Abb. 15).

Insgesamt leidet die Labrumdiagnostik an einer hohen Variabilität bei Normalpersonen. Insbesondere anterior-superior werden häufig Varianten angetroffen. Das Foramen sublabrale findet sich in $\mathbf{1 2} \%$ der asymptomatischen Bevölkerung und ist MR-tomographisch gekennzeichnet durch einen Flüssigkeits- bzw. Kontrastmitteldurchtritt zwischen superiorem Labrum und Glenoidoberrand [28]. Eine weitere Variante im oberen anterioren Labrum stellt der so genannte $>$ Buford-Komplex dar [28]. Er ist durch ein fehlendes Labrum anterior-superior gekennzeichnet und mit einer Verdickung des mittleren glenohumeralen Ligaments assoziiert (• Abb. 16). Diese Konstellation findet sich in 1,5\% der asymptomatischen Bevölkerung.

Neben den anatomischen Varianten gilt es zu bedenken, dass arthroskopisch normal erscheinende Labrumstrukturen MR-tomographisch in nur 50\% das zu erwartende Normalbild aufweisen (hypointens, dreiecksförmig und glatt begrenzt) [34]. Viele der arthroskopisch normal beschriebenen Labren sind abgerundet, diffus signalalteriert oder zeigen kleine Konturirregularitäten. Zur Differenzierung zwischen pathologischen Veränderungen und Normvarianten muss die Lokalisation der Läsion mitberücksichtigt werden: Anterior-inferiore Veränderungen stellen häufig pathologische Veränderungen dar, während anterior-superiore Veränderungen häufig Ausdruck von Normvarianten sind.

\section{Knorpelläsionen}

Sie können sowohl im Zusammenhang mit Rotatorenmanschettenläsionen als auch mit Labrumläsionen vorgefunden werden. Bei Patienten mit einer Impingementsymptomatik beträgt die Prävalenz von glenohumeralen Knorpelläsionen 33\% [10]. Die MR-Arthrographie ist zur Beurteilung des glenohumeralen Knorpels nur mäßig geeignet. Knorpelläsionen unmittelbar neben Labrumläsionen werden als GLAD-Läsionen („glenolabral articular disruption") bezeichnet (• Abb. 17) [14]. 


\section{Schlussbemerkung}

Die MRT der Schulter spielt neben Anamnese und klinischer Untersuchung eine bedeutende Rolle bei der Diagnose und Differenzierung zahlreicher Schulterpathologien und der Abklärung chronischer Schulterschmerzen. Wichtige Indikationsgebiete der MRT sind die Evaluation der Rotatorenmanschette und des Labrum-Kapsel-Apparats. Besondere Bedeutung hat die Beurteilung der Rotatorenmanschettenmuskulatur. Die immer noch kontrovers diskutierte MR-Arthrographie zeigt ihr größtes Potenzial bei der präzisen Unterscheidung von kleinen Rotatorenmanschettenläsionen, Labrum-Kapsel-Läsionen und Läsionen der langen Bizepssehne.

\section{Korrespondierender Autor \\ PD Dr. M. Zanetti}

Radiologie, Universitätsklinik Balgrist, Forchstraße 340, 8008 Zürich, Schweiz

E-Mail: marco.zanetti@balgrist.ch

Interessenkonflikt: Es besteht kein Interessenkonflikt. Der korrespondierende Autor versichert, dass keine Verbindungen mit einer Firma, deren Produkt in dem Artikel genannt ist, oder einer Firma, die ein Konkurrenzprodukt vertreibt, bestehen. Die Präsentation des Themas ist unabhängig und die Darstellung der Inhalte produktneutral.

\section{Literatur}

1. Binkert CA, Zanetti M, Hodler J (2001) Patient's assessment of discomfort during MR arthrography of the shoulder. Radiology 221: 775-778

2. Bitzer M, Nasko M, Krackhardt T et al. (2004) [Direct CT-arthrography versus direct MR-arthrography in chronic shoulder instability: comparison of modalities after the introduction of multidetector-CT technology]. Rofo 176: 1770-1775

3. Bjorkenheim JM (1989) Structure and function of the rabbit's supraspinatus muscle after resection of its tendon. Acta Orthop Scand 60: 461-463

4. Bui-Mansfield LT, Taylor DC, Uhorchak JM, Tenuta JJ (2002) Humeral avulsions of the glenohumeral ligament: imaging features and a review of the literature. AJR Am J Roentgenol 179: 649-655

5. Chandnani VP, Gagliardi JA, Murnane TG et al. (1995) Glenohumeral ligaments and shoulder capsular mechanism: evaluation with MR arthrography. Radiology 196: 27-32

6. Emig EW, Schweitzer ME, Karasick D, Lubowitz J (1995) Adhesive capsulitis of the shoulder: MR diagnosis. AJR Am J Roentgenol 164: 1457-1459

7. Erickson SJ, Cox IH, Hyde JS et al. (1991) Effect of tendon orientation on MR imaging signal intensity: a manifestation of the "magic angle" phenomenon. Radiology 181: 389-392

8. Fuchs B, Weishaupt D, Zanetti M et al. (1999) Fatty degeneration of the muscles of the rotator cuff: assessment by computed tomography versus magnetic resonance imaging. J Shoulder Elbow Surg 8: 599-605

9. Goutallier D, Postel JM, Bernageau J et al. (1994) Fatty muscle degeneration in cuff ruptures. Preand postoperative evaluation by CT scan. Clin Orthop Rel Res 304: 78-83

10. Guntern DV, Pfirmann CW, Schmid MR et al. (2003) Articular cartilage lesions of the glenohumeral joint: diagnostic effectiveness of MR arthrography and prevalence in patients with subacromial impingement syndrome. Radiology 226: 165-170

11. Mengiardi B, Pfirrmann CW, Gerber C et al. (2004) Frozen shoulder: MR arthrographic findings. Radiology 233: 486-492
12. Monu JU, Pruett S, Vanarthos WJ, Pope TL Jr (1994) Isolated subacromial bursal fluid on MRI of the shoulder in symptomatic patients: correlation with arthroscopic findings. Skeletal Radiol 23: 529-533

13. Neviaser TJ (1993) The anterior labroligamentous periosteal sleeve avulsion lesion: a cause of anterior instability of the shoulder. Arthroscopy 9: 17-21

14. Neviaser TJ (1993) The GLAD lesion: another cause of anterior shoulder pain. Arthroscopy 9: 22-23

15. Newberg AH, Munn CS, Robbins AH (1985) Complications of arthrography. Radiology 155: 605-606

16. Nové-Josserand L, Gerber C, Walch G (1997) Lesions of the antero-superior rotator cuff. LippincottRaven, Philadelphia

17. Pfirrmann CW, Zanetti M, Weishaupt D et al. (1999) Subscapularis tendon tears: detection and grading at MR arthrography. Radiology 213: 709-714

18. Schulte-Altedorneburg G, Gebhard M, Wohlgemuth WA et al. (2003) MR arthrography: pharmacology, efficacy and safety in clinical trials. Skeletal Radiol 32: 1-12

19. Snyder SJ, Karzel RP, Del Pizzo W et al. (1990) SLAP lesions of the shoulder. Arthroscopy 6: 274-279

20. Strobel K, Pfirrmann CW, Zanetti M et al. (2003) MRI features of the acromioclavicular joint that predict pain relief from intraarticular injection. AJR Am J Roentgenol 181: 755-760

21. Strobel K, Hodler J, Meyer DC et al. (2005) Fatty atrophy of supraspinatus and infraspinatus musc les: accuracy of US. Radiology 237: 584-589

22. Vahlensieck M, Sommer T, Textor J et al. (1998) Indirect MR arthrography: techniques and applications. Eur Radiol 8: 232-235

23. Walch G, Nove-Josserand L, Levigne C, Renaud E (1994) Tears of the supraspinatus tendon with ,hidden "lesions of the rotator cuff interval. J Shoulder Elbow Surg 3: 353-360

24. Waldt S, Burkart A, Lange P et al. (2004) Diagnostic performance of MR arthrography in the assessment of superior labral anteroposterior lesions of the shoulder. AJR Am J Roentgenol 182: 12711278
25. Weishaupt D, Zanetti M, Tanner A et al. (1999) Lesions of the reflection pulley of the long biceps tendon. MR arthrographic findings. Invest Radiol 34: 463-469

26. Wiley AM (1991) Arthroscopic appearance of frozen shoulder. Arthroscopy 7: 138-143

27. Willemsen UF, Wiedemann $\mathrm{E}$, Brunner $\mathrm{U}$ et al. (1998) Prospective evaluation of MR arthrography performed with high-volume intraarticular saline enhancement in patients with recurrent anterior dislocations of the shoulder. AJR Am J Roentgenol 170: 79-84

28. Williams MM, Snyder SJ, Buford D Jr (1994) The Buford complex - the "cord-like" middle glenohumeral ligament and absent anterosuperior labrum complex: a normal anatomic capsulolabral variant. Arthroscopy 10: 241-247

29. Wischer TK, Bredella MA, Genant HK et al. (2002) Perthes lesion (a variant of the Bankart lesion): MR imaging and MR arthrographic findings with surgical correlation. AJR Am J Roentgenol 178: 233-237

30. Yu JS, Ashman CJ, Jones G (2002) The POLPSA lesion: MR imaging findings with arthroscopic correlation in patients with posterior instability. Skeletal Radiol 31: 396-399

31. Zanetti M, Gerber C, Hodler J (1998) Quantitative assessment of the muscles of the rotator cuff with MR imaging. Invest Radiol 33: 163-170

32. Zanetti M, Weishaupt D, Gerber C, Hodler J (1998) Tendinopathy and rupture of the tendon of the long head of the biceps brachii muscle: evaluation with MR arthrography. AJR Am J Roentgenol 170: 1557-1561

33. Zanetti M, Jost B, Hodler J, Gerber C (2000) MR imaging after rotator cuff repair: full-thickness defects and bursitis-like subacromial abnormalities in asymptomatic subjects. Skeletal Radiol 29: 314319

34. Zanetti M, Carstensen T, Weishaupt D et al. (2001) MR arthrographic variability of the arthroscopically normal glenoid labrum: qualitative and quantitative assessment. Eur Radiol 11:559-566 
Bitte beachten Sie:

Antwortmöglichkeit nur online unter: CME.springer.de Die Frage-Antwort-Kombinationen werden online

Welche Antwort zur MR-Arthrographie der Schulter trifft zu?

$\square$ Gadoliniumhaltiges Kontrastmittel wird unverdünnt zur i. v. Konzentration direkt ins Gelenk injiziert.

$\square$ Die Injektionsmenge beträgt maximal $5 \mathrm{ml}$.

$\square$ Die Injektionsmenge erhöht sich nach rezidivierenden Schulterluxationen.

$\square$ Die Injektion des Kontrastmittels wird als unangenehmer empfunden als das Liegen im MR-Scanner.

$\square$ Die Infektionsrate bei diagnostischer Arthrographie ist hoch.

\section{Welche Antwort zu Rotatoren-} manschettenläsionen trifft zu?

$\square$ Die am häufigsten lädierte Sehne ist die des Subskapularismuskels.

$\square$ Superior-posteriore Läsionen sind eher degenerativ als posttraumatisch bedingt.

$\square$ Magic-angle-Artefakte sind bei Sequenzen mit langen Echozeiten (>37 ms) nachzuweisen.

$\square$ Partialrupturen der Supra- und Infraspinatussehne treten nur artikularseitig auf.

$\square$ Supskapularissehnenrupturen werden häufig in Ober- und Unterrandläsionen unterteilt.

Ab welcher Größe gilt eine
Kapselverdickung als Hinweis für
ein schmerzhaftes AC-Gelenk?
$1 \mathrm{~mm}$.
$3 \mathrm{~mm}$.
$5 \mathrm{~mm}$.
$7 \mathrm{~mm}$.
$9 \mathrm{~mm}$.

Welche Antwort zur Atrophie und Verfettung der Rotatorenmanschette trifft nicht zu?

$\square$ Im Tierexperiment wurden Atrophie und Verfettung schon nach 4 Wochen beobachtet.

$\square$ Die Verfettung der Rotatorenmanschettenmuskulatur wird häufig nach der Goutallier-Klassifikation eingeteilt.

$\square$ Die Goutallier-Klassifikation kann nicht für die Beurteilung der Verfettung der Rotatorenmanschette im CT verwendet werden.

$\square$ Zur Beurteilung der Atrophie der Supraspinatusmuskulatur wird das Tangentenzeichen herangezogen.

$\square$ Die Beurteilung der Rotatorenmanschettenmuskulatur beschränkt sich im Ultraschall auf Supraspinatus-, Infraspinatus- und Teres-minorMuskulatur.

\section{Welche Antwort trifft nicht zu?} Flüssigkeitsansammlungen in der Bursa subdeltoidea/subacromialis können assoziiert sein mit......

$\square$ transtendinösen Rupturen.

$\square$ Bursitis.

$\square$ Rotatorenmanschettenimpingement mit Tendinopathie der Supraspinatussehne.

$\square$ Labrumläsion.

$\square$ Os acromiale.

\section{Welche Antwort zur langen Bizepssehne ist richtig?}

Läsionen der langen Bizepssehne treten nie isoliert auf.

$\square$ Bei Rupturen der Subskapularissehne kann es nie zu Beeinträchtigungen der langen Bizepssehne kommen.

$\square$ Die lange Bizepssehne kann eine Partial- oder Totalruptur aufweisen, aber keine Tendinopathie. $\square$ Am häufigsten sind die Läsionen der langen Bizepssehne im Rotatorenmanschettenintervall anzutreffen.

$\square$ Die komplette lange Bizepssehnenruptur wird bevorzugt auf den parakoronalen Aufnahmen diagnostiziert.

\section{Welche Antwort trifft zu?} SLAP-Läsionen werden...

$\square$ definiert als Läsion des superioren Labrums mit a.-p. Ausdehnung.

$\square$ eingeteilt nach Snyder, basierend auf 6 Typen.

$\square$ beim Vorliegen von Typ I als instabil bezeichnet.

$\square$ beim Vorliegen eines dislozierten Labrumrisses als Typ-II-Läsion klassifiziert.

$\square$ einfacher diagnostiziert beim Vorhandensein eines physiologischen Sulcus zwischen superiorem Labrum und hyalinem Knorpel des Glenoids.

Welche Antwort zu den glenohumeralen Ligamenten trifft zu?

$\square$ Das inferiore glenohumerale Ligament ist bei den so genannten PoulieLäsionen lädiert.

$\square$ Eine Poulie-Läsion ist charakterisiert durch Signalintensitätserhöhungen am Subskapularissehnenoberrand.

$\square$ Ein Kriterium für das Vorliegen einer Poulie-Läsion ist eine laterale Subluxation der langen Bizepssehne auf axialen Aufnahmen.

$\square$ Bei vorderen Schulterluxationen ist das mediale glenohumerale Ligament am häufigsten rupturiert.

$\square$ Am häufigsten reißen die glenohumeralen Ligamente an der humeralen Insertion aus und werden als GLAD-Läsion bezeichnet. 
Welche der folgenden Faktoren spielen vermutlich keine bedeutende Rolle bei der Diagnose einer "frozen shoulder"?

$\square$ Pathologische Veränderungen im Rotatorenmanschettenintervall.

$\square$ Verdickung der Gelenkkapsel breiter als $6 \mathrm{~mm}$.

$\square$ Pathologische Veränderungen im Recessus axillaris.

$\square$ Verdickung des korakohumeralen Ligaments.

$\square$ Obliteration des subkorakoidalen Fettgewebes.

\section{Welche der folgenden Antworten} trifft zum Labrum glenoidale nicht zu?

$\square$ Das normale Labrum ist im MRT scharf begrenzt, dreieckförmig und weist ein hypointenses Signal auf.

$\square$ Bei der klassischen Bankart-Läsion bleibt das Periost intakt.

$\square$ Die Arthro-CT vermag ossäre Glenoidrandverletzungen besser abzugrenzen als die Arthro-MR.

$\square$ Das sublabrale Foramen wird im MRT in etwa $12 \%$ der asymptomatischen Bevölkerung gefunden.

$\square$ Der so genannte Buford-Komplex ist gekennzeichnet durch ein fehlendes anterior-superiores Labrum und mit einer Verdickung des mittleren glenohumeralen Ligaments assoziiert.

Diese Fortbildungseinheit ist 12 Monate auf CME.springer.de verfügbar.

Den genauen Einsendeschluss erfahren Sie unter CME.springer.de.

\title{
Hier steht eine Anzeige.
}

\author{
算 Springer
}


Hier steht eine Anzeige.

刑 Springer 